\title{
Contraintes, Potentialités Et Opportunités Sociales Et Économiques De La Filière Anacarde (Côte d'Ivoire)
}

\section{Gnénémon Tuo}

Université Alassane Ouattara (UAO) de Bouaké, Côte d'Ivoire

Doi:10.19044/esj.2019.v15n13p206 URL:http://dx.doi.org/10.19044/esj.2019.v15n13p206

\section{Résumé}

Depuis quelques décennies, le Nord de la Côte d'Ivoire est confronté à une dynamique de précarisation sociale et économique du fait de nombreux paramètres (crises sociopolitiques, changement climatique, sécheresses, volatilité des prix agricoles etc.). Cependant, l'une des opportunités socioéconomiques qui est la filière anacarde se trouve confrontée à des mutations particulières portées par la crise socio-politique qu'a connue le pays. Dans un tel contexte de vulnérabilité où le système de commercialisation intérieure présente de nombreuses contraintes, une démarche a été proposée dans cette étude. Il s'agissait de comprendre et d'expliquer les changements induits par le délitement de la filière anacarde pendant la crise socio-politique, tout en identifiant les contraintes, les potentialités et les opportunités. L'étude repose sur des notes d'observations participantes, des entretiens semi-structurés, des enquêtes et une revue documentaire. Ainsi, les crises sociopolitiques de 2002 et postélectorale de 2010 ont fait intervenir de nouveaux acteurs, de nouvelles zones d'ombres dans la filière anacarde. Face à ces modifications, les producteurs et les acheteurs se sont adaptés par la mise en place de stratégies.

Mots clés: Anacarde, commercialisation, contraintes, opportunités ; potentialités. 


\title{
Constraints, Potentialities and Social and Economic Opportunities of the Cashew Nut Sector (Ivory Coast)
}

\author{
Gnénémon Tuo \\ Université Alassane Ouattara (UAO) de Bouaké, Côte d'Ivoire
}

\begin{abstract}
In recent decades, the north of Côte d'Ivoire has been confronted with a dynamic of social and economic precariousness due to many parameters (socio-political crises, climate change, droughts, volatility of agricultural prices etc.). However, one of the socio-economic opportunities that is the cashew industry is facing particular changes brought by the socio-political crisis in the country. In such a context of vulnerability where the internal marketing system presents many constraints, an approach has been proposed in this study. The aim was to understand and explain the changes brought about by the disintegration of the cashew nut sector during the socio-political crisis, while identifying constraints, potentialities and opportunities. The study is based on participant observation notes, semi-structured interviews, surveys and a literature review. Thus, the socio-political crises of 2002 and postelection of 2010 brought in new actors, new areas of shadow in the cashew nut sector. In the face of these changes, producers and buyers have adapted by putting strategies in place.
\end{abstract}

Keywords: Cashew nut; marketing; constraints; opportunities; potential.

Depuis quelques décennies, l'agriculture ivoirienne subit des chocs récurrents de plus en plus forts et de plus en plus divers (crises socio-politique, sécheresse, volatilité des prix agricoles etc.) sous l'effet de changements globaux majeurs (climatique, économique, social, politique, environnemental, ...) (Gnénémon, 2018). Dans le même temps, les actions réciproques notamment les stratégies et les politiques de recherche et de développement agricoles ont montré leurs limites : résultats mitigés, des politiques d'ajustement structurel agricole, du désengagement de l'État des services d'appui à l'agriculture etc.

De fait, le Nord de la Côte d'Ivoire est une zone géographique particulière. Elle est confrontée à une dynamique de précarisation en raison de nombreux paramètres dont l'une des opportunités économiques qui est la 
filière anacarde se trouve confrontée à des mutations particulières portées par les différentes crises socio-politiques. Plus qu'une activité économique, la commercialisation de l'anacarde a façonnée les producteurs. Cette opportunité économique a eu son sens en l'absence d'alternative économique encadrée comme cela a été le cas dans la zone forestière avec le binôme café-cacao, les plants palmier et les plants hévéa.

Cependant, l'anacardier est une (sinon la seule) culture pérenne sur laquelle les structures de développement ont le moins investi en Côte d'Ivoire. N'ayant bénéficié d'engagement spécifique de l'État, cette culture s'est construite autour de la capacité des producteurs à se structurer. Aujourd'hui, la filière anacarde recèle des potentiels et des opportunités dont la profitabilité est nécessairement liée à une bonne politique de commercialisation intérieure et extérieure et à la transparence des coûts pour une rémunération équitable des acteurs. Mais, ces potentialités et opportunités font face à des contraintes générées par le délitement de la filière.

La présente étude est donc un essai d'identification des contraintes, des potentialités et des opportunités majeures de la commercialisation de l'anacarde en Côte d'Ivoire de 2002 à 2015. Bien que le circuit de commercialisation soit long et complexe, cette activité joue non seulement un rôle clé dans l'économie des producteurs mais également dans l'intégration réelle de la filière anacarde dans l'économie nationale.

\section{Question et Méthode}

Cette étude vise à comprendre et à expliquer les changements induits par le délitement de la filière anacarde pendant la crise socio-politique, tout en identifiant les contraintes, les potentialités et les opportunités. Autrement dit, quelles sont les contraintes, les potentialités et les opportunités inhérentes générées par le délitement du circuit de commercialisation de l'anacarde ?

Afin de répondes à cette question de recherche, une collecte des données empiriques primaires de cette étude s'est effectuée dans cinq districts. Ce sont les districts de Savane (la région du Poro et du Tchologo) avec 20,50\% de la production nationale, la Vallée du Bandama (la région du Hambol) avec $21,85 \%$ de la production nationale, du Woroba (la région du Béré) avec $10,57 \%$ de la production nationale et du Zanzan (la région du Gontougo) avec $25,40 \%$ de la production nationale et le district d'Abidjan (principalement la ville d'Abidjan où s'y est déroulé l'essentiel de la recherche documentaire).

Durant plusieurs phases exploratoires de 2013 à 2015, deux cent dix (210) producteurs d'anacarde et trente-sept (37) acheteurs (pisteurs, commerçants, grossistes, exportateurs) localisés dans trente-neuf (39) villes et villages ont été interrogés. Des observations, des entretiens semi-structurés, des enquêtes par questionnaire, des interviews individuelles ont été menés auprès de différents acteurs de la filière, des discussions formelles, des 
dépouillements d'articles, l'écoute de supports enregistrés, la consultation de site web et une revue bibliographique sur la commercialisation des produits issus de l'anacardier ont été faits. Le tout, pour identifier les contraintes à la bonne performance du marché, les potentialités et les opportunités de la filière.

Problématique de la chaîne de valeur du circuit de commercialisation des noix de cajou

Mapping des acteurs qui interviennent dans la commercialisation de l'anacarde

Le schéma 1 montre les différents acteurs essentiels de la chaîne de commercialisation des noix brutes de Côte d'Ivoire. Comme le montre ce schéma, le processus de mise en marché fait intervenir une multitude d'opérateurs et d'acteurs. Généralement, les transactions entre producteurs, pisteurs, commerçants et autres acteurs sont fonction de la qualité, de la quantité et du prix du produit. Dans le contexte de crise, la commercialisation des noix brutes de cajou s'est fait sans contrôle, ni des structures de la filière, ni de l'État. Ce manque de contrôle a fait du circuit de commercialisation, un circuit peu structuré et peu maîtrisé avec des acteurs qui ne sont pas formellement identifiés.

Schéma 1 : Structure du circuit de commercialisation des noix brutes de 2002 à 2012

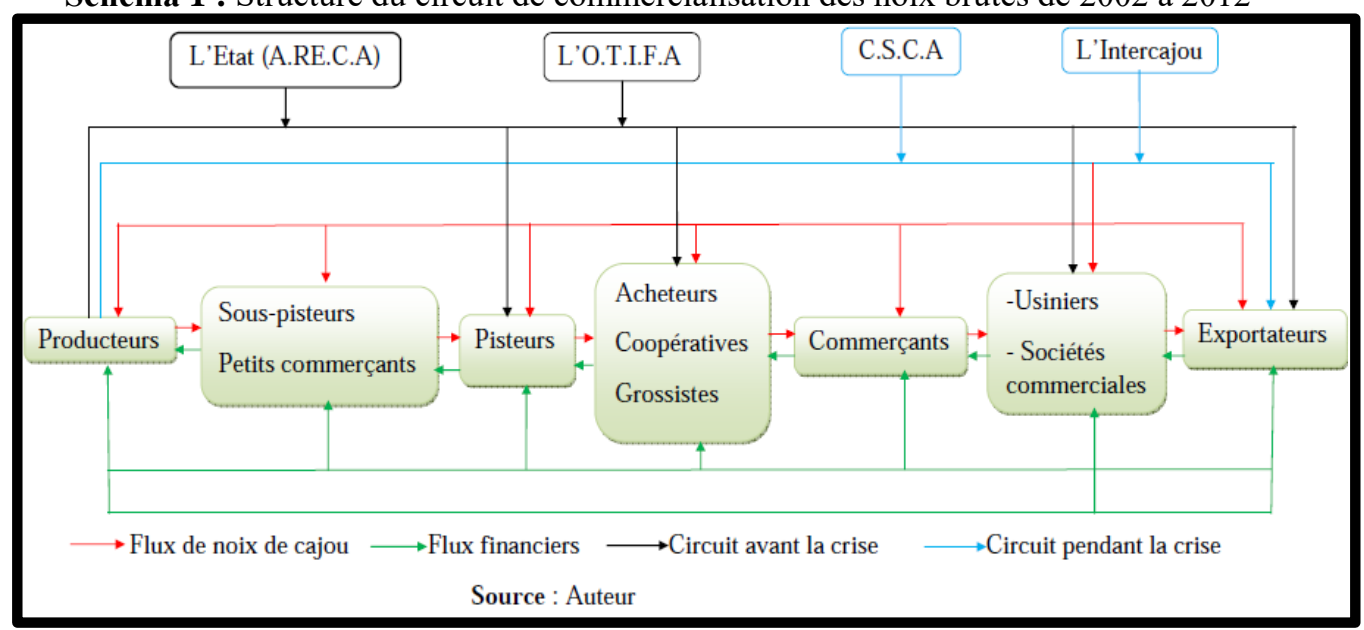

Source : Données d'enquête, 2013, 2014, 2015

Chacune des structures du schéma 1 exerce des fonctions dans la chaîne de commercialisation. Quelles sont ces fonctions ? 
Schéma 2 : Fonctions de chaque structure

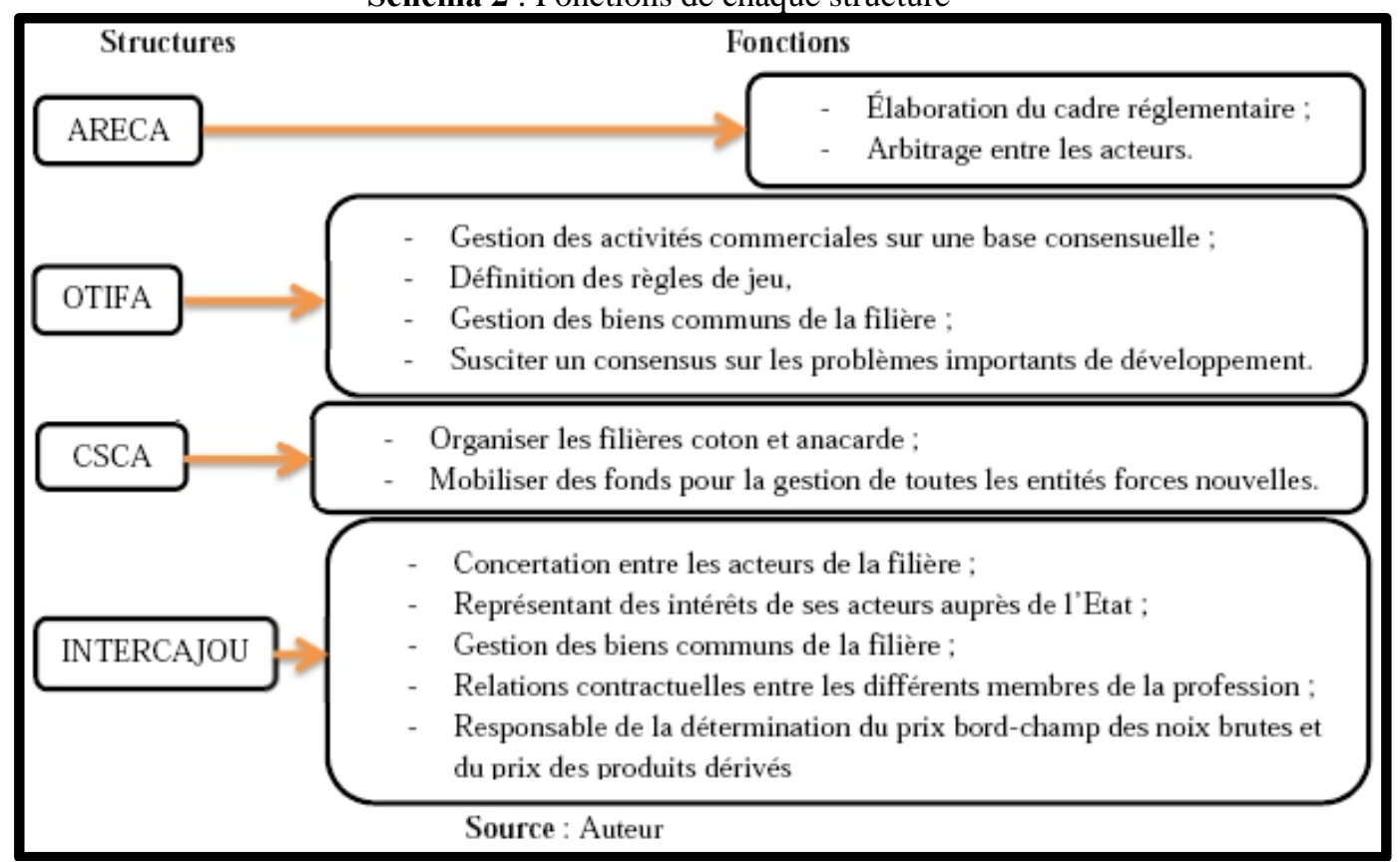

Source : Données d'enquête, 2013, 2014, 2015

De ces quatre structures du schéma 2, seule l'Autorité de Régulation du Coton et de l'Anacarde (ARECA) existait avant la crise de 2002. Les trois autres ont été créées pendant la crise. L'Intercajou est né en remplacement de l'Organe Transitoire Interprofessionnelle de la Filière Anacarde (OTIFA). Les relations entre ces structures de gestion de la filière étaient des relations personnalisées. Aucune de ces structures n'avait les moyens d'étendre ses pouvoirs sur toute la zone de production. La zone contrôlée par les Forces Nouvelles (FN) était gérée par le Comité de Suivi Coton et Anacarde (CSCA). Le reste de la Zone était sous la responsabilité de l'ARECA (représentant de l'État). Seule l'Intercajou exerçait sur tout le territoire sans un réel pouvoir.

L'absence de service de contrôle en amont et en aval du commerce de la noix de cajou entre les producteurs et les intermédiaires, les usiniers, les sociétés commerciales puis les exportateurs (cf. schéma 3) bien avant et pendant la crise socio-politique de 2002 a été cruciale pour les producteurs. Ils ont subi la loi des intermédiaires et autres acteurs qui achetaient les noix brutes à des prix dérisoires. Quelles sont les contraintes perçues par chaque acteur du circuit de commercialisation? 
Schéma 3 : Fonctions de chaque acteur

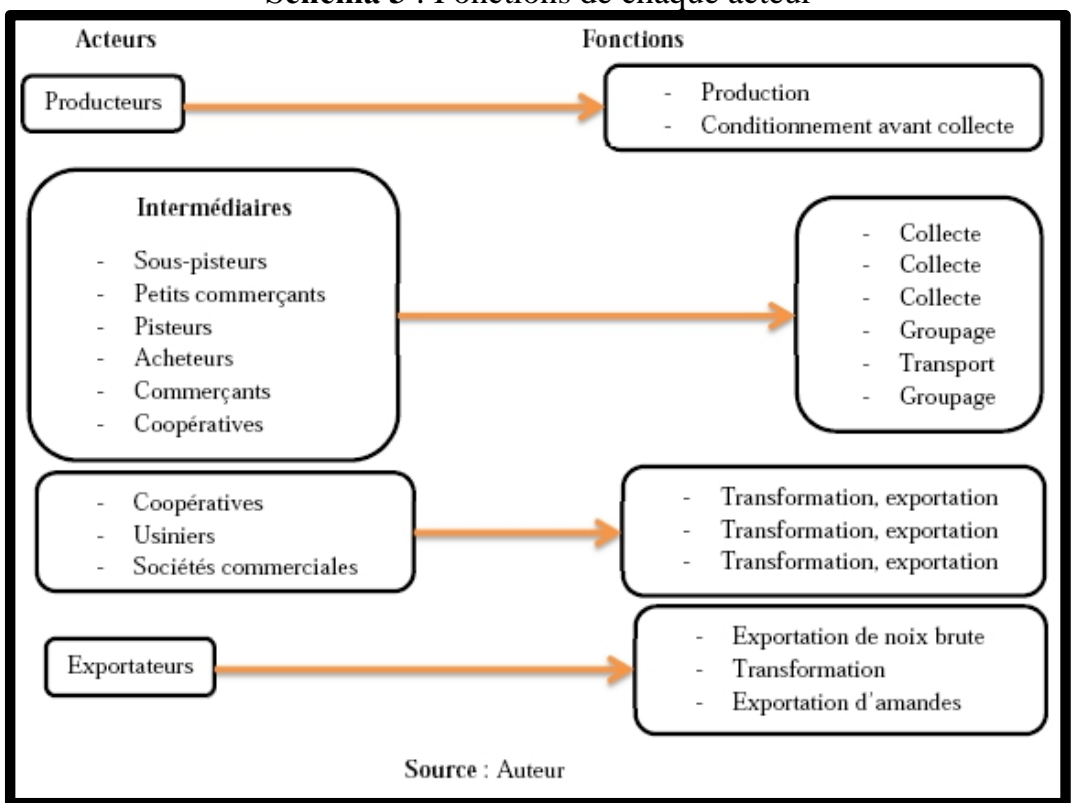

Source : Données d'enquête, 2013, 2014, 2015

\section{Mapping des acteurs qui interviennent dans la commercialisation} intérieure des noix de cajou à partir 2013

Depuis 2013, on a assisté progressivement à la disparition de certains acteurs qui exercent dans la commercialisation des noix brutes de cajou. Le schéma suivant montre les différents acteurs qui interviennent depuis 2013 dans la commercialisation des noix brutes de cajou :

Schéma 4: Acteurs du circuit de commercialisation intérieure de la noix de cajou à partir de 2013

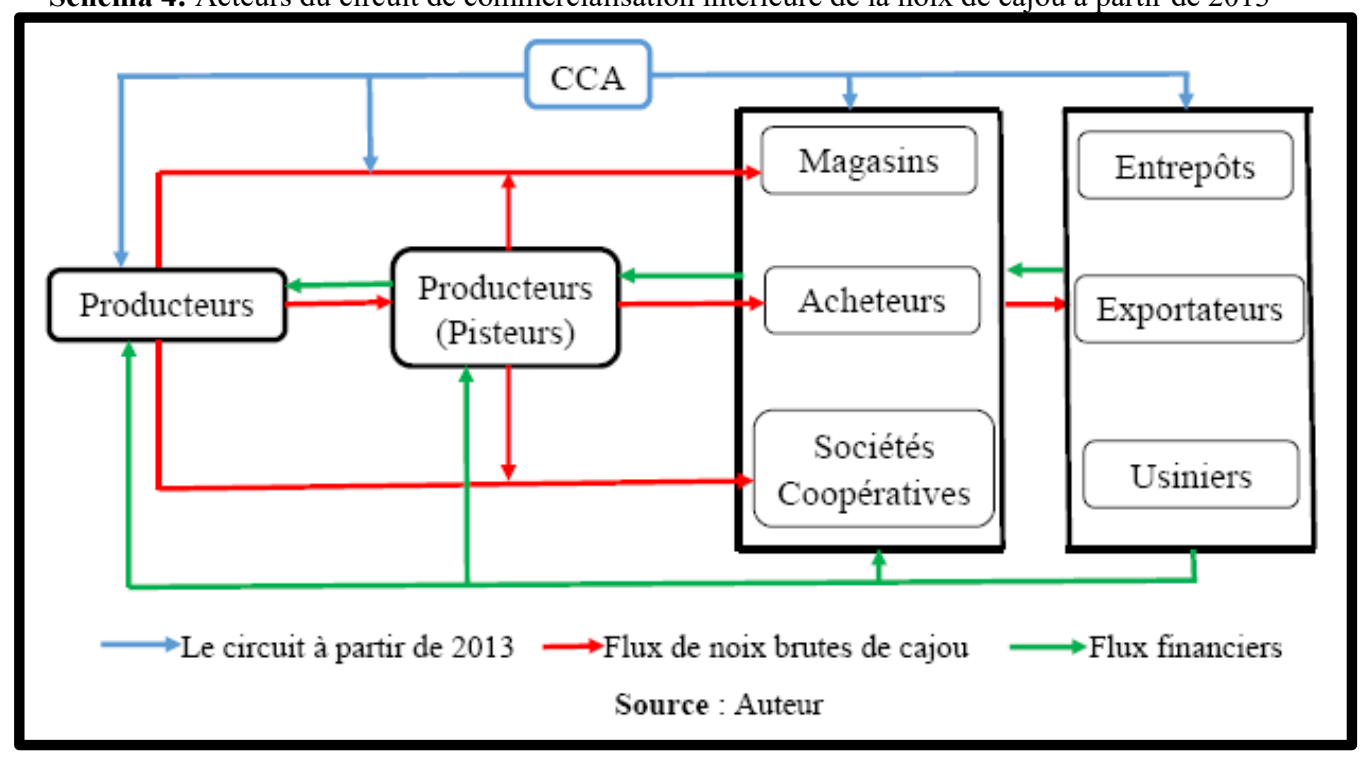


Suite aux réformes dans la filière anacarde à partir de 2013, le schéma 4 , nous présente quatre groupes d'acteurs qui interviennent dans le circuit de commercialisation des noix brutes. Or, le schéma 1 que nous avons vu plus haut, présentait 7 groupes d'acteurs qui intervenaient dans le circuit avant la nouvelle réforme. Dans le schéma 1 , on avait 4 structures de gestion qui sont intervenues dans la filière concomitamment ou successivement. Le schéma 4 ne présente que le CCA qui est la seule structure (représentant l'État) qui gère la filière.

Dans ce schéma 4, un groupe d'acteurs exerce illégalement. Il s'agit des producteurs qui sont devenus des pisteurs ou acheteurs de noix brutes de cajou. En effet, ce groupe d'acteurs est localisé dans les villages. Ils achètent les noix auprès de leurs collègues producteurs avant et après le lancement de la campagne pour ensuite les revendre plus tard à la hausse. Après les différentes réformes, plusieurs acteurs ont disparu de l'arène du circuit de commercialisation des noix brutes de cajou. Il s'agit des :

- sous-pisteurs ;

- petits commerçants ;

- commerçants ;

- sociétés commerciales.

Dans l'arène des structures de gestion de la filière anacarde, on constate également la disparition de l'INTERCAJOU et du Comité de Suivi du Coton et de l'Anacarde (structure créée par les Forces Nouvelles). Ce qui a rendu le circuit de commercialisation des noix brutes plus court. Yuna (2010) pense qu'en terme de potentiel, les enjeux du circuit court de commercialisation sont décrits comme considérables, puisque, outre la captation de valeur en faveur du producteur, il permet la réalisation d'économies sur les autres segments de la chaîne de coûts. C'est un état de fait que nous soutenons.

\section{Contraintes perçues par chaque acteur du mapping}

Face à la complexité du circuit de commercialisation pendant la crise, les producteurs et les acheteurs ont évoqué plusieurs contraintes tant au niveau de la production que de la commercialisation. Bien que complexe, la commercialisation des noix de cajou unit les producteurs aux exportateurs. En effet, il existe plusieurs intermédiaires entre les producteurs et les exportateurs. Ce qui nous intéresse ici, ce n'est pas l'individu en tant que personne physique, mais ce qui est extérieur et s'impose à lui (Durkheim, 1988).

La plupart des problèmes soulevés par les acteurs interrogés ont été identifiés comme causes principales du délitement de la filière anacarde. Nous allons relever celles qui constituent des contraintes majeures. Ces contraintes varient selon l'acteur. 


\section{- Au niveau des producteurs}

- Faible organisation des producteurs et du marché local

Notre enquête menée auprès des producteurs montre que le niveau des ventes groupées reste très faible. Les statistiques suivantes montrent l'appartenance ou non des producteurs à un groupement ou une organisation de producteurs :

Appartenance à un groupement/organisation de producteurs

Tableau 1 : Les interventions sous forme de groupement/organisation des producteurs de noix de cajou

\begin{tabular}{|c|c|c|c|c|c|}
\hline \multicolumn{2}{|l|}{} & Effectifs & Pourcentage & $\begin{array}{c}\text { Pourcentage } \\
\text { valide }\end{array}$ & $\begin{array}{c}\text { Pourcentage } \\
\text { cumulé }\end{array}$ \\
\hline Valide & OUI & 2 & 1,0 & 1,0 & 1,0 \\
\hline & NON & 208 & 99,0 & 99,0 & 100,0 \\
\hline & Total & 210 & 100,0 & 100,0 & \\
\hline
\end{tabular}

Source : Données d'enquête, 2013, 2014, 2015

Le tableau 1 montre que $1 \%$ des 210 producteurs interviewés appartient à un groupement ou coopérative de producteurs. Au-delà de ces coopératives de faible taux de regroupement des producteurs, un autre groupe de personnes a fait surface ces dernières années dans le domaine de la commercialisation de l'anacarde, il s'agit des groupements informels. Ils sont la cause des interactions entre les individus des groupements formels.

Dès qu'ils y a un groupe formel, il y a des groupes informels qui se créent à cause des interactions entre les individus. Une fois formés, ces groupes ont leur propre personnalité et développent leur mode de fonctionnement. On peut dire qu'il existe deux types de groupements informels : les groupements d'intérêt et les groupements d'amitié. Par ailleurs, les résultats du tableau 2 montrent que les producteurs préfèrent la vente collective.

Tableau 2 : Le choix du modèle de vente le plus avantageux selon les producteurs

\begin{tabular}{|c|c|c|c|c|c|}
\hline \multicolumn{6}{|c|}{ Modèle de vente préféré } \\
\hline & & Effectifs & Pourcentage & $\begin{array}{c}\text { Pourcentage } \\
\text { valide }\end{array}$ & $\begin{array}{c}\text { Pourcentage } \\
\text { cumulé }\end{array}$ \\
\hline \multirow{3}{*}{ Valide } & Collective & 149 & 71,0 & 71,0 & 71,0 \\
\hline & Individuelle & 61 & 29,0 & 29,0 & 100,0 \\
\hline & Total & 210 & 100,0 & 100,0 & \\
\hline
\end{tabular}

Source : Données d'enquête, 2013, 2014, 2015

Le tableau 2 montre que $71 \%$ des 210 producteurs interviewés préfèrent la vente collective. Cela montre la volonté affichée des producteurs de se regrouper en coopérative. Le faible taux de regroupement vient du fait qu'ils ne seront plus libres de vendre quand ils voudront et à qui ils voudront. C'est surtout le fait que la coopérative ne peut pas répondre aux besoins pressants (besoin de liquidité monétaire) d'un membre. Chatou (2016) parle 
de faible efficacité opérationnelle de ces coopératives car elles sont incapables de répondre aux besoins de leurs adhérents. Les efforts d'organisations des acteurs sont louables, avec des initiatives pertinentes. Mais toutes les organisations (de producteurs d'anacarde) sont encore fragiles car récentes. De plus il y a une mésentente entre les leaders des producteurs et une faible capacité des organisations de producteurs à mobiliser ces derniers. L'ambiance observée en avril-mai 2018 ne garantit pas encore la création effective de l'interprofession à très court termes (Jeske \& Konnon, 2018). Le Collectif Stratégies Alimentaires (CSA, 2014) pense donc qu'il est essentiel de redéfinir ce qu'est une coopérative, s'interroger sur le modèle, peut-être le repréciser et ne pas penser uniquement en termes de compétition, mais également en termes de coopération.

- La chaîne de commercialisation est trop longue

L'inorganisation des acteurs notamment des producteurs fait que n'importe qui intervient sans en avoir la compétence ni les autorisations légales. À cela s'ajoute l'abus de confiance des collecteurs indépendants, des commerçants grossistes des grandes villes et le non-respect des engagements et des poids déclarés lors des transactions. Outre les producteurs, le circuit de commercialisation des noix de cajou comme décrit plus haut, est animé par une série de contrevenants non identifiés.

- Méconnaissance des techniques de contrôle de la qualité

La plupart des producteurs n'ont jamais été formés aux différentes techniques de contrôle de la qualité. Au moment de la commercialisation des noix brutes, certains acheteurs refusent d'acheter la production au prix fixé, d'où, un des facteurs de variabilité du prix d'achat des noix brutes.

\section{- L'analphabétisme des producteurs}

Les producteurs de 'l'or brun" sont très souvent grugés par les pisteurs et autres acheteurs lors de la pesée des noix, du fait qu'ils ne savent pas lire et écrire. Ce qui fait d'eux des potentiels perdants ${ }^{13}$. En effet, lors de la pesée des noix, les acheteurs cherchent toujours à se faire des marges bénéficiaires en annonçant souvent de faut poids aux producteurs qui sont analphabètes.

\section{- Manque de bascules appropriées}

Les bascules utilisées par la plupart des pisteurs et autres acheteurs ne sont pas appropriées. Certaines personnes notamment les pisteurs les règles de façon à gagner plus de poids au cours des achats. Les producteurs analphabètes sont victimes de cette forme de fraude.

\section{- Négociation des prix}

\footnotetext{
${ }^{13} \mathrm{Vu}$ que les producteurs ne savent pas lire, les pisteurs et autres acheteurs usent de cette naïveté pour annoncer des poids au kilogramme qui sont souvent loin des réalités.
} 
Les producteurs n'ont aucune idée de leurs coûts de production, ce qui constituerait, selon eux, un handicap lors des transactions sur les marchés. Par ailleurs, l'inorganisation des producteurs, qui pour la plupart exercent individuellement, les défavorise dans le cadre des négociations de prix.

\section{- Variabilité du prix d'achat des noix brutes}

La variabilité du prix d'achat est pour les producteurs un élément qui concourt à l'instabilité du processus de commercialisation du produit. D'un acheteur à un autre et d'un pisteur à un autre le prix d'achat varie. Au port, l'exportateur ne respecte pas les prix fixés. On a aussi la délivrance non contrôlée des agréments d'exportation qui, entraîne de façon logique le nonrespect des prix fixés. Finalement, les producteurs sont obligés de chercher le meilleur acheteur pour vendre leurs productions. Montagne (1960) pense que les producteurs, victimes d'une constance dégradation de leurs revenus, veulent vendre plus cher leurs produits, et qu'il leur est conseillé de partir à la conquête de leurs marchés.

- Le problème de conservation des noix brutes

Les producteurs ont signalé trois principales contraintes par rapport à la conservation des noix :

$\checkmark$ un faible niveau d'équipement et le manque d'infrastructures de stockage ;

$\checkmark$ les sacs en jute sont coûteux. Ce qui les oblige à utiliser des sacs en plastique ;

$\checkmark$ la non maîtrise de la période de vente des stocks constitués pour une vente commune.

\section{- Financement des producteurs}

Les contraintes de financement constituent le facteur limitant qui a été très largement évoqué par les producteurs. Ce facteur est dû, dans plusieurs cas, à une méconnaissance des conditions d'obtention de crédits bancaires. La méconnaissance du système bancaire fait que certains producteurs prennent des engagements de prêts périlleux auprès de certains commerçants ou pisteurs.

- Le manque d'encadrement technique des producteurs

Les producteurs dans leur majorité ne bénéficient pas d'encadrement de la part des structures d'encadrement. Seules quelques coopératives de producteurs bénéficient de l'encadrement technique et du suivi de la part de certaines institutions non gouvernementales. Ce manque d'encadrement des producteurs est un facteur limitant dans l'amélioration de la production et de la qualité des noix brutes.

\section{- Amélioration de la qualité}

L'insuffisance des semences améliorées et le manque d'équipement post-récolte constituent des handicaps pour l'amélioration de la qualité de la production. Dans le cadre du développement de la transformation et de 
l'exportation, l'industrie ivoirienne aura besoin d'une matière première de qualité.

\section{- Contraintes extérieures aux producteurs}

Les producteurs estiment que les acheteurs leurs imposent certaines contraintes. Il s'agit notamment du prix d'achat bord champ et la qualité des noix. La fixation du prix indicatif qui se faisait par l'Intercajou dont ils étaient membres, se faisait souvent sans eux.

- La dépendance totale sur l'Inde pour l'achat des noix brutes de cajou

Ces dernières années (depuis 2006) ont montré à travers les stocks invendus, le danger que court la production ivoirienne en comptant sur un seul client, en l'occurrence l'Inde. La Côte d'Ivoire a souvent des quantités importantes de noix invendues parce que les indiens ne les ont pas achetés.

\section{- Au niveau des sous-pisteurs}

Ce maillon du circuit de commercialisation est confronté au problème de financement, à la qualité des noix brutes (la récolte de noix immatures et le non séchage des noix hypothèquent de plus en plus la qualité des noix et des amandes) et à l'instabilité du prix d'achat. Les techniques de contrôle de la qualité sont non connues par les sous-pisteurs et sont peu performantes.

\section{- Au niveau des petits commerçants}

Le problème de financement est général pour tous les acheteurs. La particularité des petits commerçants est le manque de bascules appropriées et le problème de conservation des noix.

\section{- Au niveau des pisteurs}

Dans ce maillon important du circuit de commercialisation, l'on a relevé le problème de financement, de l'instabilité des prix, de la qualité des noix et le non-respect des engagements des sous-pisteurs.

\section{- Au niveau des acheteurs}

Comme tous leurs précédents, les acheteurs sont confrontés aux problèmes de financement, à l'instabilité des prix, à la vente anticipée et la récolte des noix immatures qui de plus en plus hypothèquent la qualité des noix. On note aussi la méconnaissance des techniques de contrôle qualité.

\section{- Au niveau des coopératives}

La plupart des coopératives n'ont pas d'objectifs clairs. Certaines se sont fixé plusieurs objectifs à atteindre à la fois ce qui les déroutent souvent. Aujourd'hui, plusieurs coopératives existent de nom parce qu'elles n'ont pas pu atteindre les nombreux objectifs qu'elles s'étaient assignés.

\section{- Au niveau des grossistes}

- L'accès aux financements

Les grossistes eux rencontrent certains problèmes tels que l'accès difficile aux crédits et leurs coûts élevés et la faiblesse des capacités de 
stockage notamment dans les villages et même dans les villes des zones de production.

\section{- L'instabilité des prix}

L'instabilité des prix est un souci particulier pour les grossistes car, ils achètent des milliers de tonnes de noix de cajou brute. La volatilité des prix et l'instabilité des marchés agricoles constituent des phénomènes essentiels en agriculture (Kroll et al., 2010).

\section{- Les tracasseries routières}

Les tracasseries en l'occurrence les barrages érigés par les Forces Nouvelles pendant les crises entre les zones de collecte et les marchés puis des marchés d'échanges aux différents ports sont les principales contraintes évoquées par les grossistes. Il faut aussi noter les problèmes liés à l'information sur l'offre et la demande et la difficulté d'obtenir des volumes suffisants de bonne qualité pour servir des clients plus exigeants.

- La méfiance entre les grossistes et les pisteurs

Certains pisteurs ne respectent pas souvent leurs engagements vis-àvis de leurs financiers (les grossistes). Certains d'entre eux disparaissent avec l'argent qu'ils reçoivent pour l'achat de noix brutes. Cette situation handicape certains financiers qui sont obligés de payer les noix à de bas prix pour rattraper la perte.

\section{- Au niveau des commerçants}

Ce maillon a enregistré comme contraintes essentielles, l'instabilité des prix d'achat, la vente anticipée des noix immatures (qui de plus en plus joue sur la qualité de la production), la méconnaissance des techniques de contrôle de la qualité et le problème de transport.

\section{- Au niveau des exportateurs individuels ou sociétés}

Les problèmes essentiels de ce maillon sont la parafiscalité, les coûts d'exploitation et la cherté du port. Le président du comité national de l'Alliance africaine de cajou (ACA) de Côte d'Ivoire a dénoncé des prélèvements énormes dans la filière cajou :

«(...), il y a ce qu'on appelle le Droit unique de sortie (DUS), qui est de l'ordre de 10000 F.CFA la tonne donc 10 F.CFA le Kilo, et les parafiscalités pour certaines structures qui interviennent dans la commercialisation, comme l'Areca et le FIRCA. Les taxes tournent globalement autour de 16500 FCFA la tonne, donc 16,50 F.CFA par Kilo. C'est énorme par rapport aux autres pays comme le Ghana, le Burkina Faso et le Nigeria où il n'y a pratiquement pas de taxe à l'exportation de la noix de cajou. En plus de cela, en Côte d'Ivoire, nos coûts d'exploitation sont plus élevés à cause de la cherté du Port. Ce qui fait que nous sommes mieux-disant en termes d'offres, par rapport au Ghana ou au Togo, ce qui encourage la fuite des produits vers le Ghana. (... )» (Koffi Yao Appia, 21 Août 2013). 


\section{- Au niveau des transformateurs locaux}

Concernant les transformateurs, il existe plusieurs contraintes dont les plus astreignants sont :

\section{- La qualité}

Les noix de cajou de mauvaises qualités ne sont pas profitables pour les transformateurs locaux. La bonne qualité des amandes dépend de la bonne qualité des noix de cajou (l'outturn, le taux d'humidité et le taux de grainage).

- Le financement

Le financement est l'un des facteurs clés qui déstabilisent facilement les transformateurs locaux. La plupart des échecs de transformation enregistrées depuis les premières tentatives de transformation des noix de cajou en Côte d'Ivoire sont le fait d'un manque de financements adéquats.

\section{- L'équipement pour la transformation}

Selon les transformateurs, l'équipement nécessaire à la transformation est extrêmement coûteux et les taxes de dédouanement aussi. Le matériel de transformation des noix brutes en amandes est encore peu performant. Le taux de transformation reste encore très faible (moins de 10\%).

- Amélioration de la qualité

À terme, l'exportation des noix de cajou doit respecter la norme NI 4595 : 2009 élaborée par CODINORM. Cette norme classifie les noix de cajou en trois grades avec des critères liés à la teneur en eau, au rendement en amandes (Outturn). En dehors de ces trois grades, les noix ne doivent pas être autorisées à l'exportation. Elles doivent donc être transformées localement ou retraitées.

De façon générale la transformation requiert des matières premières de bonne qualité. L'équipement de décorticage dans ces zones n'est pas suffisamment performant (perte élevée, qualité faible, problème de matériel de rechange, problème de qualité du carburant).

\section{- Financement}

Pour les Petites et Moyennes Entreprises (PME), la non-habitude de travailler avec les banques constitue un handicap majeur. C'est le cas de la coopérative "Chongagnigui de Karakoro" qui a vu les portes de son unité de décorticage fermées du fait d'un manque de financement.

\section{- Équipement}

Il faut des équipements pour transformer la production et mieux les vendre. Le sous-entendu est que les produits transformés rapportent plus. Si l'on peut comprendre qu'un produit transformé comprend plus de valeur ajoutée, il reste à savoir comment est répartie cette valeur ajoutée.

- L'emballage et l'étiquetage pour un bon marketing

L'emballage et l'étiquetage jouent un rôle important dans l'après production pour un bon marketing et une meilleure commercialisation. Ils jouent pour beaucoup dans la valeur ajoutée. Aujourd'hui, les consommateurs 
sont devenus de plus en plus exigeants sur la qualité et le bon état sanitaire du produit. L'emballage aussi doit protéger, transporter, informer et attirer. Et comme l'affirment souvent les spécialistes, l'emballage est l'agent commercial muet du fabricant. Il est donc important d'emballer le produit de façon attirante mais aussi solide.

\section{Potentialités perçues au niveau national et mondial}

Malgré l'importance des contraintes qui réduisent les performances du système de commercialisation des noix de cajou, les acteurs de la filière, principalement ceux du circuit de commercialisation disposent de potentialités qui, bien valorisés, permettraient d'améliorer le commerce des produits issus de l'anacardier et d'améliorer le revenu de tous les acteurs.

\section{$\checkmark$ Potentialités au niveau national}

Les potentialités générées au niveau national sont nombreuses. Dans cet article, nous n'allons pas faire une liste complète de ces potentialités. Cependant, on note qu'une grande partie des communautés vivant dans les zones de production, sont frappées soit par la pauvreté monétaire et/ou par la pauvreté non monétaire critique. La filière anacarde pourrait donc constituer un potentiel de financement du développement local et de redressement du revenu des ménages de ces localités.

L'anacardier étant une essence forestière, son rôle dans la protection de l'environnement est quasi évident dans la mesure où son intégration aux systèmes de culture permet de lutter contre la destruction du couvert végétal et par conséquent la dégradation physique des terres. Les systèmes à base d'anacarde s'accommodant bien pendant les cinq (5) premières années avec des cultures aussi dégradantes du sol telles que le coton et l'igname constituent l'une des alternatives d'inversion des tendances de désertification des espaces.

Les produits issus de l'anacardier représentent 2,9\% des exportations totales en valeur de la Côte d'Ivoire en 2012, ce qui place l'anacarde au 6ème rang des produits exportés (au niveau SH-6) après le cacao, le caoutchouc et les huiles de pétrole, et avant le coton (PACIR, 2013). Comme annoncé, il existe de nombreuses potentialités entre autres :

\section{- La réduction de la pauvreté}

$\mathrm{Au}$ vu des avantages que procure la vente des noix brutes aux producteurs, les règles de mise en marché des noix, si elles sont respectées, peuvent permettre d'augmenter leur revenu et donc de contribuer à la réduction de la pauvreté. Mais au regard des problèmes identifiés, le système semble encore avoir des jours difficiles à connaître si l'on ne règle pas les problèmes ci-après :

$>$ le problème de non-respect du prix d'achat ;

$>$ le problème de la mise en place de l'Interprofession de la filière anacarde ; 
$>$ le problème de la qualité du produit.

- La sécurité alimentaire

La vente des noix de cajou arrive dans une période (période de soudure) où les producteurs n'ont plus d'autres produits à vendre. L'argent que procure cette vente des noix permet aux producteurs de s'acheter pour certains du riz, du maïs, de l'igname pour la consommation quotidienne et pour d'autres, scolariser les enfants etc.

\section{$\checkmark$ Potentialités au niveau mondial}

Depuis 2012, la Côte d'Ivoire est le deuxième pays producteur mondial et premier exportateur mondial de noix de cajou. Il y a quelques années, l'Inde était le seul pays importateur des noix ivoirienne. Aujourd'hui, on compte en plus de l'Inde, le Brésil et la Chine sur la liste des principaux importateurs de noix de cajou.

\section{Opportunités perçues par les différents acteurs}

La filière anacarde ivoirienne regorge des opportunités qui sont diverses, que ce soit au niveau de la production, de la commercialisation que de la transformation de l'anacarde. La filière offre des opportunités d'emplois aussi bien au niveau des plantations qu'en dehors de celles-ci. Comme exemple, la coque de cajou qui dérive de la transformation de l'amande de cajou offre des opportunités d'ajout de valeur (iCA, 2010). Elle contient selon le même auteur un acide toxique, le cashew nut sell liquide (CNSL) ou baume qui est utilisé comme additif dans des applications industrielles. Ce secteur de la transformation offre une création d'emplois additionnels assez rémunérés et majoritairement occupés par des femmes. Deuxième producteur d'anacarde au monde après l'Inde et premier exportateur mondial de noix brute, la Côte d'Ivoire offre des opportunités d'investissements avec pour objectif de transformer $100 \%$ de sa production de noix de cajou en 2020. La transformation de la pomme en vin, en jus et autres liqueurs qui n'est pas encore fortement exploitée, offre des opportunités de création d'emplois.

On parle très souvent de valeur ajoutée des noix par référence à la transformation, ce qui n'est pas nécessairement juste. On peut réaliser la valeur ajoutée déjà au niveau des noix brutes avant de passer à la transformation en amandes. La valeur ajoutée des noix brutes pourrait provenir de :

$>$ l'amélioration du traitement post-récolte à travers la récolte des noix mures ;

$>$ la collecte des noix tombées par terre après une maturité complète ;

$>$ le séchage immédiat des noix après la récolte au niveau du champ et des collecteurs ;

$>$ l'emballage des noix dans des sacs de jute. 
Toutes ces possibilités constituent des opportunités de valeurs ajoutées pour les noix brutes. Cependant, celles-ci ne sont pas systématiquement pratiquées en Côte d'Ivoire au niveau des champs. Le seul effort consiste à collecter des noix mures car certains producteurs impatients cueillent souvent les noix immatures pour les sécher afin de se faire de l'argent rapidement du fait de l'accès difficile au crédit. Ce qui donne une mauvaise qualité du produit et par conséquent une mauvaise réputation pour les grossistes qui approvisionnent le marché indien.

En dépit de l'importance des contraintes qui réduisent les performances du système de commercialisation des noix brutes, les acteurs de la filière notamment ceux du circuit de commercialisation disposent d'opportunités (la diversification des revenus, les réformes institutionnelles de 2013, du Programme National d'Investissement Agricole, le réseau routier en nette amélioration, l'éligibilité de la Côte d'Ivoire à l'AGOA, la présence de structures non gouvernementales d'appui technique et financier) qui, bien saisies, permettraient d'améliorer le revenu des acteurs et la commercialisation des produits issus de l'anacardier.

\section{L'anacarde dans l'économie nationale}

La commercialisation des produits issus de l'anacardier ne profite pas seulement aux producteurs de noix de cajou. Elle profite à toutes les régions du pays. En effet, selon les statistiques faites en 2010 par l'ARECA, plus de 2,5 millions de personnes sont concernées par les bénéfices de la production des noix de cajou. Les plus grands bénéficiaires ne sont pas les producteurs. Ils sont plutôt issus des rangs des transformateurs, des pisteurs, des petits commerçants, des exportateurs et autres intervenants. Avant 2011, la plus grande usine de transformation de noix de cajou se trouvait au Sud (Abidjan) qui n'est pas une zone de production de noix de cajou et, ceux qui y travaillaient n'étaient pas forcement des personnes des zones de production.

Les exportations de noix de cajou et d'amandes de cajou se font à partir du port d'Abidjan qui n'est pas situé dans une zone de production de noix de cajou. Ce port avait perdu le flux d'exportations des produits issus de l'anacardier pendant la crise socio-politique. Le tableau 3 présente les flux financiers générés par la commercialisation des noix de cajou entre 2007 et 2015.

Les formules de calcul appliquées sont ainsi présentées :

VOLUME EXPORTE $($ en Kg $) \times$ PRIX FOB MOYEN $(F C F A)=V A L E U R$ FOB

$\operatorname{VOLUME}($ en tonne $) \times$ PRIX MOYEND'ACHAT $(F C F A)=$

GAIN PRODUCTEURS

10\% DES REVENUS DU VOLUME DES PRODUITS EXPORTES= GAIN DE L'ETAT 
Tableau 3 : Flux financiers générés par la commercialisation des noix de cajou en Côte d'Ivoire

\begin{tabular}{|cccc|}
\hline Années & Valeur FOB & Gain producteurs & Gain de l'État \\
\hline $\mathbf{2 0 0 7}$ & 51.153 Milliards & 42 Milliards & 5.115 Milliards \\
$\mathbf{2 0 0 8}$ & 47.724 Milliards & 56.1 Milliards & 4.772 Milliards \\
$\mathbf{2 0 0 9}$ & 56.25 Milliards & 57.75 Milliards & 5.625 Milliards \\
$\mathbf{2 0 1 0}$ & 76.752 Milliards & 77.52 Milliards & 7.675 Milliards \\
$\mathbf{2 0 1 1}$ & 79.22 Milliards & 110 Milliards & 7.922 Milliards \\
$\mathbf{2 0 1 2}$ & 109.2 Milliards & 101.25 Milliards & 10.92 Milliards \\
$\mathbf{2 0 1 3}$ & 165.075 Milliards & 87.5 Milliards & 16.507 Milliards \\
$\mathbf{2 0 1 4}$ & 221.340 Milliards & 169.434 Milliards & 22.134 Milliards \\
$\mathbf{2 0 1 5}$ & 333.200 Milliards & 288.029 Milliards & 39.337 Milliards \\
\hline
\end{tabular}

Source : Auteur/Données du CCA, 2015, 2016

Économiquement, l'anacarde est une alternative avérée. L'amélioration des revenus est le fait de l'augmentation de la production. La promotion de la noix de cajou permet dès lors, par les revenus générés, de parfaire les conditions de vie précaire des populations, en soignant du même coup, sa participation au PIB national. En 2012, la production était de plus de 450000 tonnes avec un chiffre d'affaires de plus 109 milliards de F.CFA. Trois (3) ans plus tard, c'est-à-dire en 2015, la production a atteint 702510 tonnes avec un chiffre d'affaires de plus de 288 milliards de F.CFA, soit plus de deux fois le chiffre d'affaires de 2012.

D'un autre point de vue, la culture de l'anacarde et sa transformation endiguent l'exode rural, sans pour autant provoquer un abandon des cultures vivrières et des petits élevages, assurant de ce fait la subsistance quotidienne. Enjeu important pour l'économie de nombreuses familles, la filière garantit l'indépendance du producteur. La diversification des produits agricoles en milieu rural ivoirien fait partie des principales stratégies adoptées par les paysans pour faire face aux contraintes alimentaires, économiques mais aussi agro-écologiques.

En effet, les producteurs espèrent arriver à améliorer leur niveau économique tout en ayant les moyens de satisfaire leurs besoins alimentaires. Outre la création d'emplois locaux, les cultures de rente permettent aux paysans de mettre un terme à l'assujettissement pendant les périodes de "soudure" où certaines trésoreries familiales se trouvent affaiblies. En général, ces cultures de rente sont des plantes non-traditionnelles introduites par les colons au travers de programmes de développement agricole initiés par l'État (Cas de l'oignon dans le Nord, puis de l'hévéa, du palmier-à-huile, du coco, ... au Sud).

Ainsi, la filière anacarde joue un rôle de plus en plus important dans l'économie de la Côte d'Ivoire. Avec le respect par endroit du prix d'achat fixé par l'État ces dernières années, ces résultats peuvent être considérés 
aujourd'hui comme remarquables contrairement à ceux obtenus les années précédentes.

\section{Conclusion}

L'anacarde est perçu aujourd'hui dans les zones de production comme un produit favorisant la résilience des acteurs du circuit de commercialisation, améliorant ainsi la condition socio-économique des acteurs in fine à la lutte contre la pauvreté. En dépit de son importance stratégique, sa contribution à la sécurité économique des acteurs, mérite d'être discutée dans le contexte d'un circuit de commercialisation qui présente des dysfonctionnements liés pour la plupart à la crise socio-politique de 2002 et à la crise post-électorale de 2010. Ces crises ont fortement contribué au délitement de la filière anacarde par la création de nombreuses contraintes et de nouvelles formes d'interactions entre les producteurs et les acheteurs. Cependant, ces crises n'ont pas seulement créé des contraintes et des formes diverses d'interactions, elles ont ouvert aussi la porte à de nombreuses potentialités et opportunités pour les producteurs et les acheteurs de l'or brun. Premier producteur et exportateur de noix brute de cajou depuis 2015 avec une ambition de transformer $100 \%$ des noix de cajou en 2020. Ces évolutions sont en grande partie le fruit d'une réforme lancée en 2013 (Jeske \& Konnon, 2018 op. cit.). Ces potentialités et opportunités sont créatrices de valeurs ajoutées et d'emplois. On retient que deux contraintes principales (la variation du prix d'achat et l'accès au crédit) sont générales pour tous les acteurs du circuit de commercialisation. L'accompagnement des acteurs à la commercialisation leur permettra de mieux planifier, de prévoir les recettes, d'anticiper les dépenses, ce qui contribuera à la sécurisation de leur revenu.

Malgré le niveau de délitement de la filière anacarde, elle regorge de plusieurs potentialités et offre par la même occasion plusieurs opportunités. Ces différentes contraintes ne peuvent avoir un apport positif que lorsqu'ils sont accompagnés d'une forte volonté politique pouvant induire des changements indispensables dans la politique agricole.

En effet, pour sortir du contexte dominé par les contraintes qui bloquent le développement de la filière anacarde, la réponse à apporter doit être éminemment politique. Et donc pour que les agriculteurs adhèrent à un quelconque modèle politique proposé par les dirigeants, ceux-ci doivent investir dans l'agriculture et dans le milieu rural d'où provient la grande partie du revenu national et surtout la grande partie de la population ivoirienne.

Bien que confronté à des contraintes au niveau de la production, de la commercialisation, de la transformation et au niveau organisationnel, la filière anacarde regorge des potentialités et des opportunités qui peuvent permettre de contourner ces contraintes. Ces potentialités et opportunités se dégagent tant au niveau de la production, de la commercialisation que de la 
transformation permettant aux producteurs et aux acheteurs de devenir de plus en résilients.

\section{References:}

1. Chatou A., 2016. L'efficacité des coopératives agricoles et le rôle des adhérents à travers leurs organisations, Lévis : Sommet international des coopératives, $26 \mathrm{p}$.

2. CSA, 2014. Les coopératives agricoles: Une réponse aux enjeux des agriculteurs familiaux? Compte-rendu du séminaire international co-organisé par le CSA, 1'UAW, la FWA et la FJA le 2 décembre 2014 à la Maison de l'Agriculture à Gemblou, Financé par la DGD, 37 p.

3. Durkheim E., 1988 [1895]. Les règles de la méthode sociologique, Paris, Flammarion

4. Gnénémon T., 2018. La filière anacarde de Côte d'Ivoire de 2002 à 2015 : entre désorganisation et jeux d'acteurs, Thèse de doctorat, Université ALASSANE OUATTARA de Bouaké, 378 p.

5. iCA, 2010. Apprécier la qualité des noix de cajou brutes, ACA/INADES Formation/TechnoServe/RONGEAD/gtz/OLAM, Manuel technique, 25p.

6. Jeske V. S. \& Konnon D-D, 2018. Capitalisation des expériences et acquis de la Côte d'Ivoire en matière de politiques publiques, de structuration et de gestion de la filière anacarde, Document de réflexion n²34, 56 p, www.ecdpm.org/dp234.

7. Kroll J-C., Aurélie T. \&\& Déruaz M., 2010. Quelle perspective de la régulation après la sortie des quotas? Faut-il encore une politique laitière européenne ? UMR 1041 INRA AGROSUP CESAER

8. Montagne R., 1960. La coopération dans le domaine de la commercialisation des produits agricoles, In : Économie rural, $\mathrm{n}^{\mathrm{0}} 44$, Commercialisation des produits agricoles, pp. $75 \quad 84$, doi : https://doi.org/10.3406/ecoru.1960.1701;

https://www.persee.fr/doc/ecoru_0013-0559_1960_num_44_1_1701.

9. PACIR, 2013. Évaluation du potentiel à l'exportation des noix de cajou en Côte d'Ivoire, U.E, ICA, Appui institutionnel et opérationnel pour l'amélioration du cadre des affaires et le renforcement de la compétitivité des exportations de la Côte d'Ivoire, Document technique $\mathrm{n}^{\mathrm{O}} \mathrm{EC}-10$ 174.E, ICT, Genève, 54p.

10. Yuna C., 2010. Formes et enjeux des circuits de commercialisation en agriculture, Sup Agro,

INRA, UMR Innovation Montpellier, $37 \mathrm{p}$. 